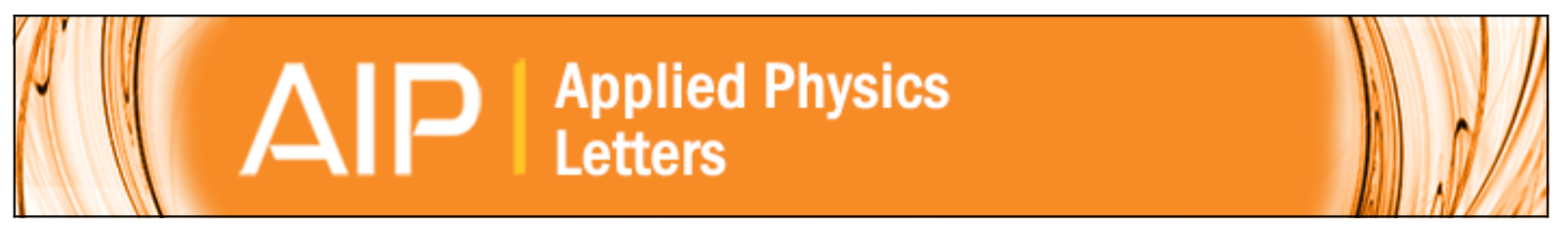

\title{
Domain formation due to surface steps in topological insulator Bi2Te3 thin films grown on Si (111) by molecular beam epitaxy
}

S. Borisova, J. Kampmeier, M. Luysberg, G. Mussler, and D. Grützmacher

Citation: Applied Physics Letters 103, 081902 (2013); doi: 10.1063/1.4818456

View online: http://dx.doi.org/10.1063/1.4818456

View Table of Contents: http://scitation.aip.org/content/aip/journal/apl/103/8?ver=pdfcov

Published by the AIP Publishing

\section{Articles you may be interested in}

Electronic structure and morphology of epitaxial Bi2Te2Se topological insulator films

J. Appl. Phys. 116, 193708 (2014); 10.1063/1.4902010

Two-dimensional weak anti-localization in $\mathrm{Bi} 2 \mathrm{Te} 3$ thin film grown on $\mathrm{Si}(111)-(7 \times 7)$ surface by molecular beam epitaxy

Appl. Phys. Lett. 102, 163118 (2013); 10.1063/1.4803018

Molecular beam epitaxy of bilayer $\mathrm{Bi}(111)$ films on topological insulator Bi2Te3: A scanning tunneling microscopy study

Appl. Phys. Lett. 101, 081603 (2012); 10.1063/1.4747715

Robust surface electronic properties of topological insulators: Bi 2 Te 3 films grown by molecular beam epitaxy Appl. Phys. Lett. 98, 222503 (2011); 10.1063/1.3595309

Epitaxial growth of Bi2Se3 topological insulator thin films on Si (111)

J. Appl. Phys. 109, 103702 (2011); 10.1063/1.3585673

Frustrated by

old technology?

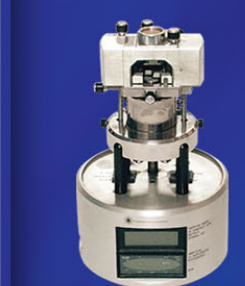

Is your AFM dead

and can't be repaired?

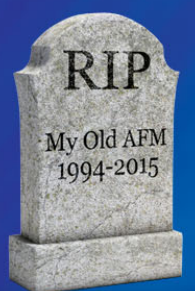

Sick of bad customer support?

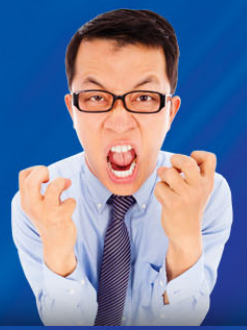

It is time to upgrade your AFM Minimum $\$ 20,000$ trade-in discount for purchases before August 31st

Asylum Research is today's technology leader in AFM 


\title{
Domain formation due to surface steps in topological insulator $\mathrm{Bi}_{2} \mathrm{Te}_{3}$ thin films grown on $\mathrm{Si}(111)$ by molecular beam epitaxy
}

\author{
S. Borisova, ${ }^{1,2}$ J. Kampmeier, ${ }^{1,2}$ M. Luysberg, ${ }^{3}$ G. Mussler, ${ }^{1,2}$ and D. Grützmacher ${ }^{1,2}$ \\ ${ }^{1}$ Peter Grünberg Institute-9, Forschungszentrum Jülich, Jülich 52425, Germany \\ ${ }^{2}$ Jülich Aachen Research Alliance, Fundamentals of Future Information Technologies, Jülich 52425, Germany \\ ${ }^{3}$ Peter Grünberg Institute-5 and Ernst Ruska-Centre for Microscopy and Spectroscopy with Electrons, \\ Forschungszentrum Jülich, Jülich 52425, Germany
}

(Received 24 April 2013; accepted 26 July 2013; published online 19 August 2013)

\begin{abstract}
The atomic structure of topological insulators $\mathrm{Bi}_{2} \mathrm{Te}_{3}$ thin films on $\mathrm{Si}$ (111) substrates grown in van der Waals mode by molecular beam epitaxy has been investigated by in situ scanning tunneling microscopy and scanning transmission electron microscopy. Besides single and multiple quintuple layer (QL) steps, which are typical for the step-flow mode of growth, a number of 0.4 QL steps is observed. We determine that these steps originate from single steps at the substrate surface causing domain boundaries in the $\mathrm{Bi}_{2} \mathrm{Te}_{3}$ film. Due to the peculiar structure of these domain boundaries the domains are stable and penetrate throughout the entire film. (C) 2013 AIP Publishing LLC. [http://dx.doi.org/10.1063/1.4818456]
\end{abstract}

Complex hexagonal layered materials such as $\mathrm{Bi}_{2} \mathrm{Te}_{3}$, $\mathrm{Bi}_{2} \mathrm{Se}_{3}, \mathrm{Sb}_{2} \mathrm{Te}_{3}$ and their alloys have recently attracted attention due to their outstanding properties as topological insulators (TIs). ${ }^{1}$ Insulating in bulk, these materials possess metallic surface states located within the bulk band gap and protected against elastic backscattering at nonmagnetic impurities by time-reversal symmetry. ${ }^{2}$ Exciting a great interest to theoretical studies, these materials present significant challenges to the experimental observation of this feature due to the unintentional bulk doping. ${ }^{3,4}$ Indeed, the crystal structure of 3D TIs is prone to a high density of antisite defects, causing a shift of Fermi energy within the whole band gap and beyond. ${ }^{5}$ This circumstance may be overcome using high quality ultrathin films in order to exclude the contribution of the bulk charge carriers or by means of counterdoping. ${ }^{6}$ Providing accurate control under the film thicknesses and the doping level, the MBE technique offers routes in order to overcome this problem. Recently, growth of perfect TI single crystalline films by MBE has been demonstrated on various substrates both highly mismatched, such as graphene, ${ }^{7} \mathrm{Si},{ }^{8,9} \mathrm{GaAs},{ }^{10}$ and nearly lattice matched, for example, $\mathrm{InP}^{11}$ or $\mathrm{BaF}_{2} \cdot{ }^{6}$ Thus, the lattice mismatch between the film and substrate is not a crucial parameter, since the growth takes place in the van der Waals growth mode..$^{12,13}$ Furthermore, the grown films are only weakly bound to the substrate and, therefore, fully relaxed. Thus, the quality of the films is not significantly influenced by strain due to the lattice mismatch, in contrast to the conventional heteroepitaxy. Besides strain-induced defects, epitaxial growth on substrates with different crystal structure may also cause the formation of antiphase domains due to the substrate steps, for example in GaAs on $\mathrm{Si}^{14-16}$ Therefore, one could assume the presence of domain boundaries in 3D TI films, affecting their electrical properties. However, both any corresponding calculations and experimental information are missing so far.

$\mathrm{Bi}_{2} \mathrm{Te}_{3}$ possesses a complicated crystal structure with a unit cell built from three so-called quintuple layers (QLs). Every QL consists of five alternating and strongly bound
$\mathrm{Bi}$ and $\mathrm{Te}$ atomic sheets in the following sequence in the [0001] direction: Te I-Bi-Te II-Bi-Te I. The coupling between the QLs is weak due to its van der Waals nature and allows layer-by-layer growth in the step-flow mode with a QL as a unit. ${ }^{17-19}$ The substrate determines the crystallographic $\mathrm{z}$ axis of the $\mathrm{Bi}_{2} \mathrm{Te}_{3}$ film perpendicular to the substrate surface. The orientation of the film matches with the $\mathrm{Si}$ substrate orientation. ${ }^{8}$ Despite the lattice mismatch of $14 \%$, the film is fully relaxed even within the 1 st QL. ${ }^{17}$ Generally, the growth on Si (111) can be separated in three phases consisting of the substrate passivation by Te, the 3D island growth, and the subsequent 2D QL step-flow mode growth of $\mathrm{Bi}_{2} \mathrm{Te}_{3}$ films without formation of any amorphous buffer layer. ${ }^{16}$ The crystal structure of $\mathrm{Bi}_{2} \mathrm{Te}_{3}$ allows the presence of two energetically equivalent twin domains. Indeed, TEM observations reveal both, vertical and horizontal domain boundaries. ${ }^{17,20,21}$ One of the twin domains can be locally overgrown by the other domain at the thickness of several $\mathrm{nm}$ and does not cause any threading or planar defects, observable at the film surface. Previously, it has been reported on a number of sub QL steps which can neither be attributed to the single unit steps usually found in the stepflow mode nor to the twin domains. However, the high dislocation density has not allowed us to determine the origin of these steps so far. ${ }^{17}$ The sub QL steps have been observed in $\mathrm{Bi}_{2} \mathrm{Se}_{3}$ films grown on Bi-covered Si (111), however, these steps disappear with increasing film thickness and their atomic structure have not been investigated. ${ }^{22}$ In this letter, we report on the atomic structure of domains due to single steps on the substrate surface, observed in topological insulator $\mathrm{Bi}_{2} \mathrm{Te}_{3}$ thin film on $\mathrm{Si}$ (111) grown in the van der Waals epitaxy mode by MBE. These domains are studied by in situ scanning tunneling microscopy (STM) and high-resolution transmission electron microscopy (HR STEM).

The MBE growth of $\mathrm{Bi}_{2} \mathrm{Te}_{3}$ films were realized in an ultra-high vacuum (UHV) chamber (base pressure $2 \times 10^{-9}$ mbar) equipped with standard effusion cells for $\mathrm{Bi}$ and $\mathrm{Te}$. Prior to the deposition, n-doped $\mathrm{Si}$ (111) substrates $(3.6-5.5 \Omega \mathrm{cm})$ have been chemically cleaned in piranha 
solution $\left(\mathrm{H}_{2} \mathrm{SO}_{4}\right.$ 2:1 $\left.\mathrm{H}_{2} \mathrm{O}_{2}\right)$ and in $1 \%$ hydrofluoric acid. The clean $\mathrm{Si}$ surface is thereby passivated by a monolayer of hydrogen. After immediate transfer to the UHV MBE a pregrowth annealing has been performed in two different ways for different samples: for the so-called low temperature annealing the substrates were heated up to $700{ }^{\circ} \mathrm{C}$ for 20 min, in order to desorb the $\mathrm{H}$ passivation layer. Alternatively, the high temperature annealing has been carried out for $30 \mathrm{~min}$ at $960^{\circ} \mathrm{C}$. The subsequent slow cool down to $700^{\circ} \mathrm{C}\left(\sim 1^{\circ} \mathrm{C} / \mathrm{s}\right)$ provides an atomically flat surface with a low roughness and density of surface defects as well as a stable $(7 \times 7)$ reconstruction, confirmed by RHEED. Atomic $\mathrm{Bi}$ and $\mathrm{Te}$ have been evaporated at the source temperatures of $\mathrm{T}_{\mathrm{Bi}}=470{ }^{\circ} \mathrm{C}, \mathrm{T}_{\mathrm{Te}}=380^{\circ} \mathrm{C}$. The growth took place under $\mathrm{Te}$ overpressure at $\mathrm{T}_{\mathrm{sub}}=300^{\circ} \mathrm{C}$, providing a high quality single crystalline $\mathrm{Bi}_{2} \mathrm{Te}_{3}$ film. ${ }^{8}$ The growth rate was $4 \mathrm{~nm} / \mathrm{h}$. Directly after $\mathrm{Bi}_{2} \mathrm{Te}_{3}$ deposition, the samples have been transferred to the in situ STM chamber under UHV conditions. The STM measurements have been performed at room temperature. Additionally, the $\mathrm{Bi}_{2} \mathrm{Te}_{3}$ films have been investigated ex situ by atomic force microscopy (AFM) and by HR STEM using an aberration corrected STEM (FEI Titan 80-300). Cross-sectional specimens were prepared by conventional grinding techniques and final ion milling with Ar ions.

Ex situ AFM measurements of $11 \mathrm{~nm}$ thick $\mathrm{Bi}_{2} \mathrm{Te}_{3}$ films show islands with diameters of $2-3 \mu \mathrm{m}$ featuring large atomically flat areas in between (see Figure 1(a)). The islands possess flat terraces with a constant width of nearly $300 \mathrm{~nm}$ separated by the single QL steps. This morphology of the film confirms the step-flow mode of growth with a QL as the growth unit. It is remarkable that every terrace replicates the shape of the previous one. Therefore, we assume that the lateral growth is accompanied by the vertical growth of the pyramids, due to the limited surface diffusion length of the adatoms. Indeed, at the moment when the width of the topmost terrace becomes larger than twice the diffusion length, a new terrace starts to nucleate on top of it. Mainly, this growth model has been used so far, in order to describe the growth of $\mathrm{Bi}_{2} \mathrm{Te}_{3}$ and related materials by thin film deposition techniques. However, a closer look at the surface
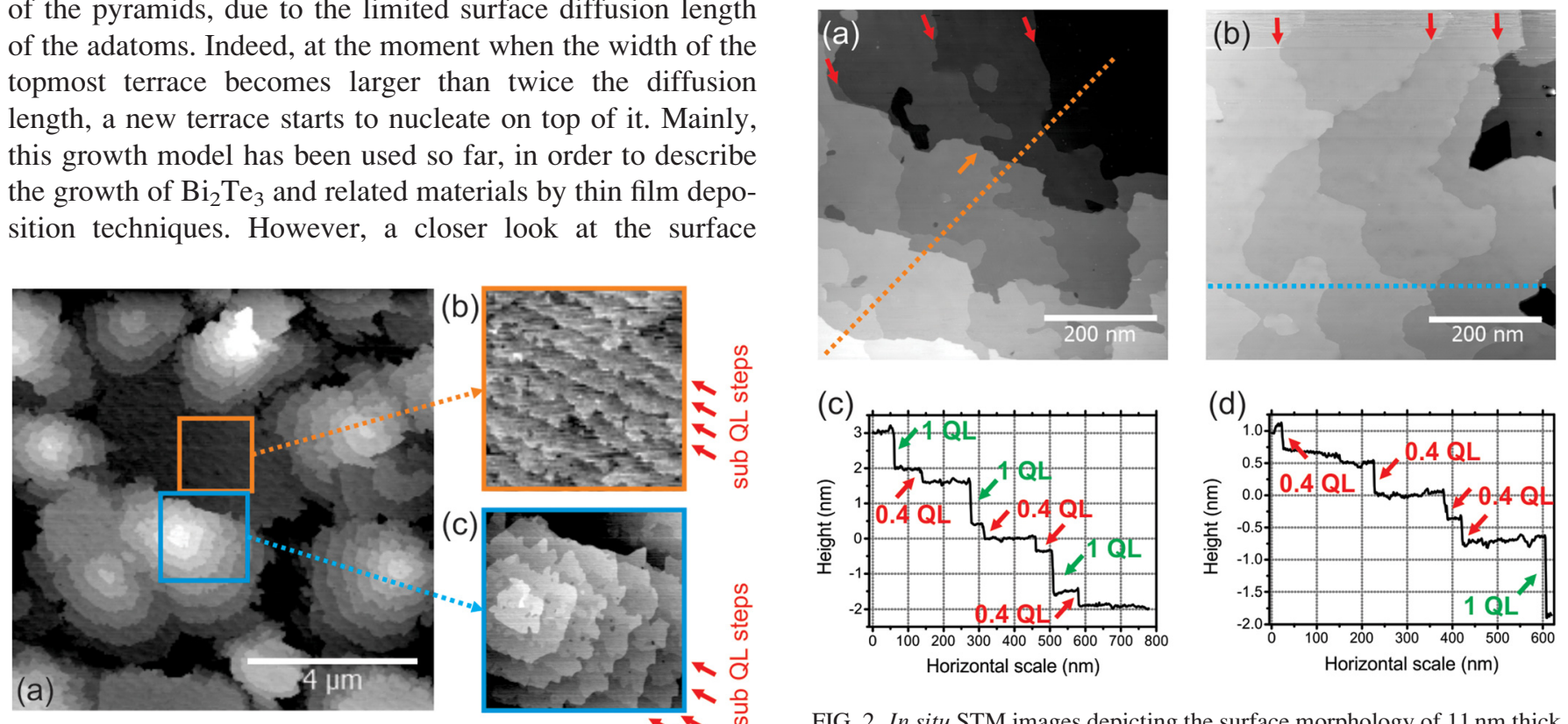

FIG. 2. In situ STM images depicting the surface morphology of $11 \mathrm{~nm}$ thick $\mathrm{Bi}_{2} \mathrm{Te}_{3}$ films grown on $\mathrm{Si}$ (111) substrates (a) overgrown after H-desorption at $700^{\circ} \mathrm{C}$, (b) in situ annealed at $960^{\circ} \mathrm{C}$ in order to obtain $(7 \times 7)$ reconstruction. (c), (d) The corresponding height profiles reveal the presence of a number of sub QL steps between the single QL steps, typically of $0.4 \mathrm{QL}$ in height. The intersection of a QL step and a 0.4 QL step (indicated by the orange arrow in (a)) demonstrates that these sub QL steps are not disturbed by the step-flow mode of growth. 
QL steps is significantly increased compared to the lowtemperature annealed substrate, the width of 0.4 QL steps is in average unchanged.

The observed morphology of the sub QL steps let us assume that the sub QL steps may originate at the substrate surface. In order to prove this assumption, the films have been studied by STM at the early nucleation stage of growth after deposition of nominally $1 \mathrm{~nm} \mathrm{Bi}_{2} \mathrm{Te}_{3}$. The atomically flat single crystalline islands have been observed at the low temperature annealed substrate (see Figure 3(a)). Due to their small size and the high density of the islands, no sub QL steps can be recognized. However, different terraces between the islands are seen. To guide the eye, colored labels are placed between the islands in the areas of the same height. The legend to the labels is shown in Figure 3(b). The labels point out the presence of 0.4 QL height difference between the neighboring terraces. Moreover, the average size of the terraces has been determined as the distance between the dotted lines in Figure 3(a), separating the areas with the predominant amounts of the certain terraces. Identically to the films of $11 \mathrm{~nm}$ thickness, the step pattern is regular, the terrace edges are parallel and the width of the terraces is about $100 \mathrm{~nm}$. Thus, the observed 0.4 QL steps replicate the substrate steps propagating throughout the entire film. The width of the $\mathrm{Bi}_{2} \mathrm{Te}_{3}$ terraces is in accordance to the distance between single steps in the Si substrate with nominally no miscut. Due to the lower density of surface defects, the surface diffusion length of the adatoms is significantly higher in case of the high temperature annealed substrate. Therefore, the nominally $1 \mathrm{~nm}$ thick film grown on the high temperature annealed substrate exhibits a low density of islands. At the same time, the 0.4 QL steps are still present at the surface (see Figure 3(c)). Thus, these steps should be attributed to
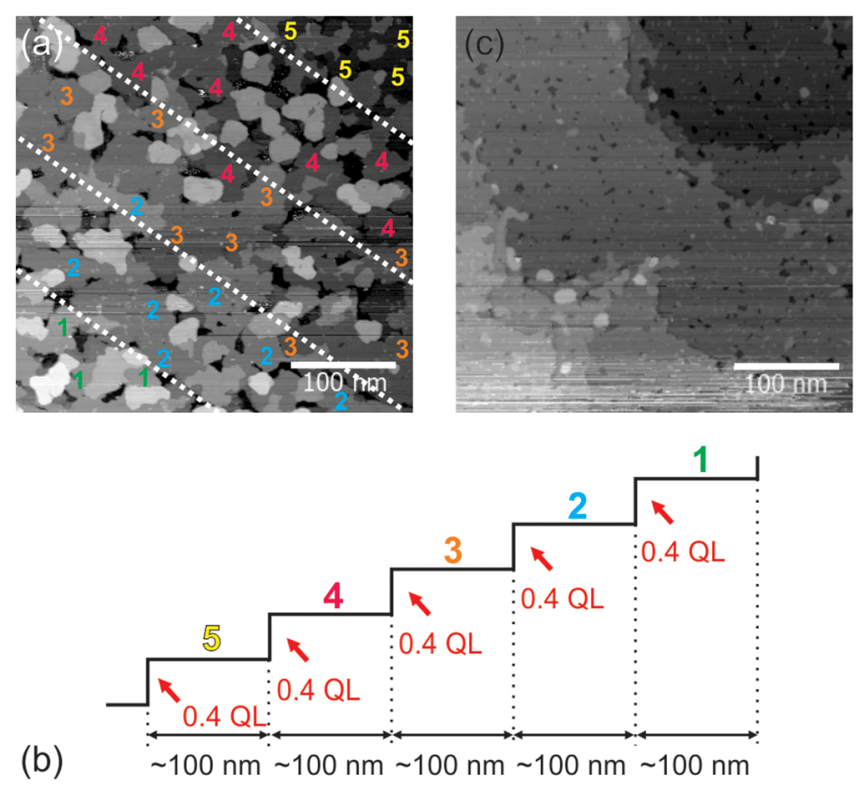

FIG. 3. (a) In situ STM image depicting the surface morphology of $1 \mathrm{~nm}$ thick $\mathrm{Bi}_{2} \mathrm{Te}_{3}$ on $\mathrm{Si}$ (111) substrate, overgrown after $\mathrm{H}$-desorption at $700^{\circ} \mathrm{C}$. The terraces are indicated by numbers from 1 to 5 for convenience (b). A regular pattern of atomically flat terraces with the width of $100-150 \mathrm{~nm}$ separated by $0.4 \mathrm{~nm}$ steps is observed. (c) Surface morphology of $1 \mathrm{~nm}$ thick $\mathrm{Bi}_{2} \mathrm{Te}_{3}$ on $\mathrm{Si}(111)$ substrate, in situ annealed at $960^{\circ} \mathrm{C}$. Terraces separated by $0.4 \mathrm{~nm}$ steps are revealed. The terraces are atomically flat and possess a small number of holes and islands compared to (a). the substrate morphology rather than to any peculiarities of the crystal structure or growth dynamics of $\mathrm{Bi}_{2} \mathrm{Te}_{3}$.

These findings have been confirmed utilizing high resolution cross sectional HR STEM. An atomically resolved image of the $\mathrm{Bi}_{2} \mathrm{Te}_{3}$ film close to the single step on a Si substrate is presented in Figure 4(a). The image displays a domain boundary, originating exactly at the substrate step and proceeding throughout the entire film. The domains at the boundary have a remarkable atomic structure: two domains are vertically shifted relative to each other by two atomic sheets (i.e., 0.4 QL). Figure 4(b) depicts the correspondent atomic model. Here, the vertical stacking sequence $(\mathrm{ABCABC} . .$.$) maintains undisturbed. Moreover, three of$ five atomic sheets match each other, whereas two of them change from Bi to Te and vice versa over the domain boundary (indicated by red triangles). Since the van der Waals gap between the QLs is vertically shifted by $0.4 \mathrm{QL}$, a $0.4 \mathrm{QL}$ is present at the film surface. Therefore, the STEM data is in good agreement with the conclusions based on the previously presented STM investigation.

(a)

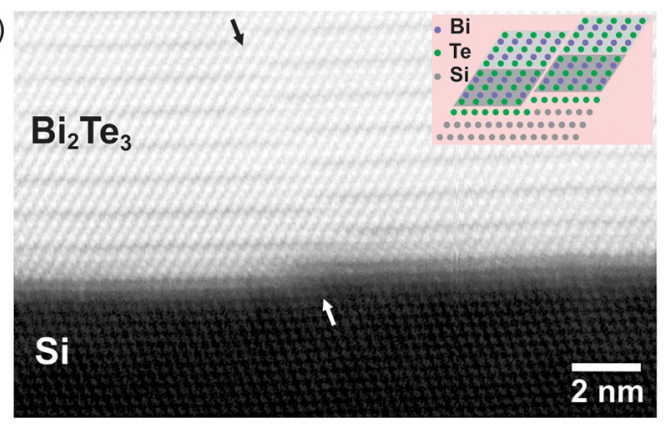

(b)

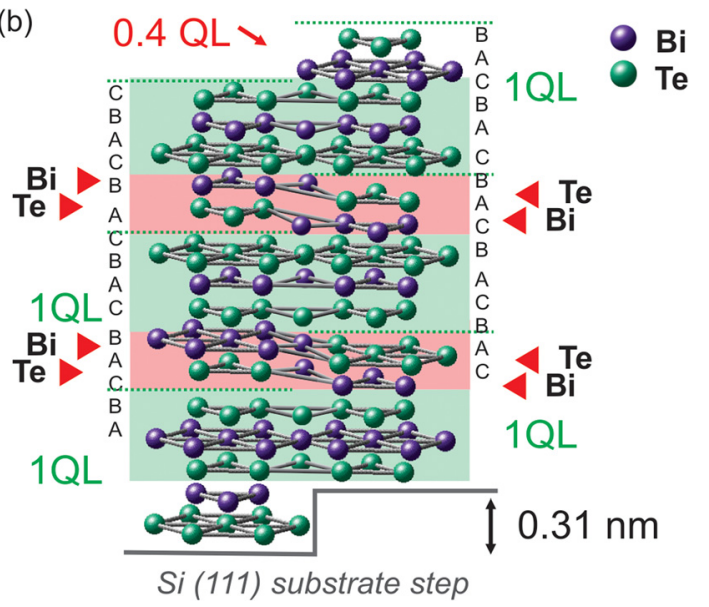

FIG. 4. (a) Cross-section of a $\mathrm{Bi}_{2} \mathrm{Te}_{3}$ film on $\mathrm{Si}$ (111) by HR STEM in (110) projection of Si. The HR STEM image indicates a domain boundary, originating at a single substrate steps. The domain boundary propagates through the complete film and causes a 0.4 QL step on the surface. The inset shows the corresponding atomic model. The stacking sequence $(\mathrm{A}, \mathrm{B}, \mathrm{C})$ of the atomic sheets remains completely undisturbed at the domain boundary. This is indicated by diagonal lines (atom rows) continuing over the boundary. (b) The crystal model explains the atomic structure of the domain boundary: three of the atomic layers of each QL (marked by green background) penetrate almost undisturbed through the domain boundary, whereas two of the atomic layers of each QL (marked by red background) change from $\mathrm{Bi}$ to $\mathrm{Te}$ and vice versa (depicted by red triangles). The QLs are indicated by dotted green lines. The stacking sequence (A, B, C) of the atomic sheets remains completely undisturbed at the domain boundary. 
In principle, these domains are similar to the antiphase domains originating at the single substrate steps, for the materials grown on substrates with the different crystal structure (particularly, lattice constants in the growth direction). The classical example is the growth of GaAs on $\mathrm{Si}^{14-16}$ Due to the complicated structure of $\mathrm{Bi}_{2} \mathrm{Te}_{3}$, the domain structure is different though. Single steps on $\mathrm{Si}$ (111) are of $0.31 \mathrm{~nm}$ in height. The step height of $0.4 \mathrm{QL}$ in $\mathrm{Bi}_{2} \mathrm{Te}_{3}$ has the best match to these steps on $\mathrm{Si}$. The observed domains are stable and the density of the domain boundaries is equal to the density of the single substrate steps. Thus, this mechanism is preferable compared to the effects provided by the weak van der Waals bonding, i.e., the easy bending of the QLs and the capability of the islands to migrate in the beginning of growth. ${ }^{17}$

In summary, our study presents domains in $\mathrm{Bi}_{2} \mathrm{Te}_{3}$ on $\mathrm{Si}$ (111), which appear due to the single steps on the Si substrate surface. The domain boundaries cause a regular pattern of parallel 0.4 QL steps observed on the surface. Combining the STM and STEM data, we ascertain that the QLs of the neighboring domains are vertically shifted by 0.4 QL. The (ABC) stacking sequence is unchanged over the boundary. However, only three atomic sheets within a QL completely match, while two other sheets change from $\mathrm{Bi}$ to $\mathrm{Te}$ and vice versa. The steps do not vanish during island coalescence. Moreover, they maintain undisturbed, while overgrown by next QLs in the step-flow mode and, hence, propagate throughout the entire film. The presence of the domains is independent of the surface preparation and, therefore, is not defined by the nucleation and growth dynamics. The study will help to understand properties of the MBE growth and substrate influence on complex TI material systems in order to improve their structural and electrical properties.

${ }^{1}$ D. Hsieh, Y. Xia, D. Qian, L. Wray, J. H. Dil, F. Meier, J. Osterwalder, L. Patthey, J. G. Checkelsky, N. P. Ong, A. V. Fedorov, H. Lin, A. Bansil, D.
Grauer, Y. S. Hor, R. J. Cava, and M. Z. Hasan, Nature (London) 460, 1101 (2009).

${ }^{2}$ M. Z. Hasan and C. L. Kane, Rev. Mod. Phys. 82, 3045 (2010).

${ }^{3}$ Y.-Y. Li, G. Wang, X.-G. Zhu, M.-H. Liu, C. Ye, X. Chen, Y.-Y. Wang, K. He, L.-L. Wang, X.-C. Ma, H.-J. Zhang, X. Dai, Z. Fang, X.-C. Xie, Y. Liu, X.-L. Qi, J.-F. Jia, S.-C. Zhang, and Q.-K. Xue, Adv. Mater. 22, 4002 (2010).

${ }^{4}$ L. Plucinski, G. Mussler, J. Krumrain, A. Herdt, S. Suga, D. Grützmacher, and C. M. Schneider, Appl. Phys. Lett. 98, 222503 (2011).

${ }^{5}$ J. Horák, J. K. Čermák, and L. Koudelka, J. Phys. Chem. Solids 47, 805 (1986).

${ }^{6}$ L. He, X. Kou, and K. L. Wang, Phys. Status Solidi (RRL) 7, 50 (2013).

${ }^{7}$ W. Dang, H. Peng, H. Li, P. Wang, and Z. Liu, Nano Lett. 10, 2870 (2010).

${ }^{8}$ J. Krumrain, G. Mussler, S. Borisova, T. Stoica, L. Plucinski, C. Schneider, and D. Grützmacher, J. Cryst. Growth 324, 115 (2011).

${ }^{9}$ H. W. Liu, H. T. Yuan, N. Fukui, L. Zhang, J. F. Jia, Y. Iwasa, M. W. Chen, T. Hashizume, T. Sakurai, and Q. K. Xue, Cryst. Growth Des. 10, 4491 (2010).

${ }^{10}$ X. Liua, D. J. Smith, H. Cao, Y. P. Chen, J. Fan, and Y.-H. Zhang, J. Vac. Sci. Technol. B 30, 02B103 (2012).

${ }^{11}$ N. V. Tarakina, S. Schreyeck, T. Borzenko, C. Schumacher, G. Karczewski, K. Brunner, C. Gould, H. Buhmann, and L. W. Molenkamp, Cryst. Growth Des. 12, 1913 (2012).

${ }^{12}$ A. Koma, Surf. Sci. 267, 29 (1992).

${ }^{13}$ A. Koma, Thin Solid Films 216, 72 (1992).

${ }^{14}$ H. Kawanami, A. Hatayama, and Y. Hayashi, J. Electron. Mater. 17, 341 (1988).

${ }^{15}$ M. Kawabe and T. Ueda, Jpn. J. Appl. Phys. Part 2 26, L944 (1987).

${ }^{16}$ N.-H. Cho and C. B. Carter, J. Mater. Sci. 36, 4209-4222 (2001).

${ }^{17}$ S. Borisova, J. Krumrain, M. Luysberg, G. Mussler, and D. Grützmacher, Cryst. Growth Des. 12, 6098 (2012).

${ }^{18}$ X. F. Kou, L. He, F. X. Xiu, M. R. Lang, Z. M. Liao, Y. Wang, A. V. Fedorov, X. X. Yu, J. S. Tang, G. Huang, X. W. Jiang, J. F. Zhu, J. Zou, and K. L. Wang, Appl. Phys. Lett. 98, 242102 (2011).

${ }^{19}$ L. He, F. Xiu, Y. Wang, A. V. Fedorov, G. Huang, X. Kou, M. Lang, W. P. Beyermann, J. Zou, and K. L. Wang, J. Appl. Phys. 109, 103702 (2011).

${ }^{20}$ D. L. Medlin, Q. M. Ramasse, C. D. Spataru, and N. Y. C. Yang, J. Appl. Phys. 108, 043517 (2010).

${ }^{21}$ D. L. Medlin and N. Y. C. Yang, J. Electron. Mater. 41, 1456 (2012).

${ }^{22}$ G. Zhang, H. Qin, J. Teng, J. Guo, Q. Guo, X. Dai, Z. Fang, and K. Wu, Appl. Phys. Lett. 95, 053114 (2009).

${ }^{23}$ R. W. G. Wyckoff, Crystal Structures, 2nd ed. (John Wiley and Sons, New York, 1964). 